\title{
A modified approach to aortic root reconstruction in children: An extended 2-patch root enlargement technique
}

\author{
Ed Peng, FRCS(CTh), and Asif Hasan, FRCS(CTh), Newcastle Upon Tyne, United Kingdom
}

\begin{abstract}
When aortic valve replacement is required in children, our favored approach is the Ross or Ross-Konno procedure. ${ }^{1,2}$ The Ross procedure may not be feasible in some settings, however, such as in patients with Marfan syndrome or previous truncus repair. In these settings, prosthetic valve replacement is required, and various annular enlargement techniques have been described to accommodate an adult rigid prosthesis in children. ${ }^{3-5}$ We have found these annular enlargement techniques to be inadequate in patients with previous aortic root surgery. We therefore describe here a modified approach to enlarge the aortic annulus, root, and proximal ascending aorta that is different from previously described techniques and demonstrate its use in 2 female pediatric patients.
\end{abstract}

\section{CLINICAL SUMMARIES}

\section{Patient 1}

The first patient had Marfan syndrome. She had undergone previous valve-sparing aortic root replacement with a 16-mm Vascutek graft (Vascutek Ltd a Terumo Company, Inchinnis, UK) at 6 years and further aortic valve repair at 8 years. At follow-up, progressive aortic stenosis and regurgitation developed.

At the age of 12 years (weight $36 \mathrm{~kg}$, body surface area $1.25 \mathrm{~m}^{2}$ ), she underwent further surgery to implant a mechanical aortic valve. Cardiopulmonary bypass was established with aortic bicaval cannulation, cooling to $32^{\circ} \mathrm{C}$, and right superior pulmonary vein venting. A transverse aortotomy was performed (Figure 1, $A$ ). The previous valve was found to be severely scarred and incompetent. The previous conduit had shrunk, and the root annulus could only accommodate a size 13-mm Hegar dilator. A Manouguian incision was performed vertically between noncoronary and left coronary cusps, extending sparingly into the roof of left atrium and the body of the anterior mitral leaflet (Figure 1,B), which allowed a size 17-mm Hegar dilator to be fitted. Another incision (Figure 1, B)

\footnotetext{
From the Department of Paediatric Cardiothoracic Surgery, The Freeman Hospital, Newcastle Upon Tyne, UK.

Disclosures: Authors have nothing to disclose with regard to commercial support.

Received for publication June 29, 2013; revisions received Aug 2, 2013; accepted for publication Aug 16, 2013; available ahead of print Sept 30, 2013.

Address for reprints: Asif Hasan, FRCS(CTh), Department of Paediatric Cardiothoracic Surgery, The Freeman Hospital, Newcastle Upon Tyne NE7 7DN, UK

(E-mail: Asif.Hasan@nuth.nhs.uk).

J Thorac Cardiovasc Surg 2013;146:1547-9

$0022-5223 / \$ 36.00$

Copyright (c) 2013 by The American Association for Thoracic Surgery

http://dx.doi.org/10.1016/j.jtcvs.2013.08.049
}

was made to enlarge the root anteriorly, incising between the left and right coronary cusps, which further accommodated a size 21-mm Carbomedics valve (Sorin Group, Arvada, Colo). Two separate counterincisions were made on the ascending aorta above the transverse aortotomy. Two separate Vascutek graft patches (Figure 1,C) were used to close the aortotomy. Each patch was fashioned to extend above the transverse aortotomy, so that it would enlarge not only the annulus but also the root and the proximal ascending aorta (Figure 2, $A$ and $B$ ). The patient came off bypass in sinus rhythm and without inotropes. Postoperative echocardiography showed good prosthetic valve and ventricular function. Her subsequent recovery was uneventful, and she was well at 2-month follow-up (Figure 2, D).

\section{Patient 2}

The second patient had previous pulmonary arterial banding at 1 month, truncus repair and truncal valve replacement at 4 months, replacement of the pulmonary arterial conduit at 2.3 years, and replacement of aortic and pulmonary conduits and enlargement of the intramural posterior coronary artery at 5 years. The Manouguian technique was used to implant a $15-\mathrm{mm}$ Shelhigh aortic conduit (Shelhigh Inc, Union, NJ).

At the age of 10 years (weight, $24 \mathrm{~kg}$; body surface area, $0.94 \mathrm{~m}^{2}$ ), the patient underwent further surgery to implant a larger aortic valve prosthesis and to replace the pulmonary conduit. The previous conduit had shrunk, and the root annulus could only accommodate a size $8-\mathrm{mm}$ Hegar dilator. A Nicks incision was performed in the mid noncoronary sinus. A further incision was made anteriorly between the right and left coronary cusps to accommodate a size 16-mm Carbomedics valve. Two separate equine pericardial patches were used to enlarge the root. The patient came off bypass in sinus rhythm on small doses of inotropes. Postoperative echocardiography showed good prosthetic valve and ventricular function. Her subsequent recovery was uneventful, and she was well at 4-month follow-up (Figure 2, E).

\section{DISCUSSION}

In the context of previous aortic surgery, a previously implanted prosthetic conduit will fix the size of the proximal aorta and additionally may show evidence of regression. A modified approach is therefore required to enlarge not only the annulus but also the aortic root and the proximal ascending aorta. In this technique, 2 separate 


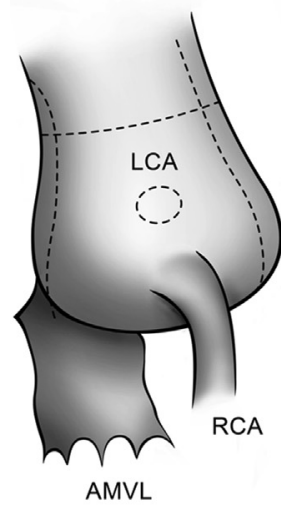

A
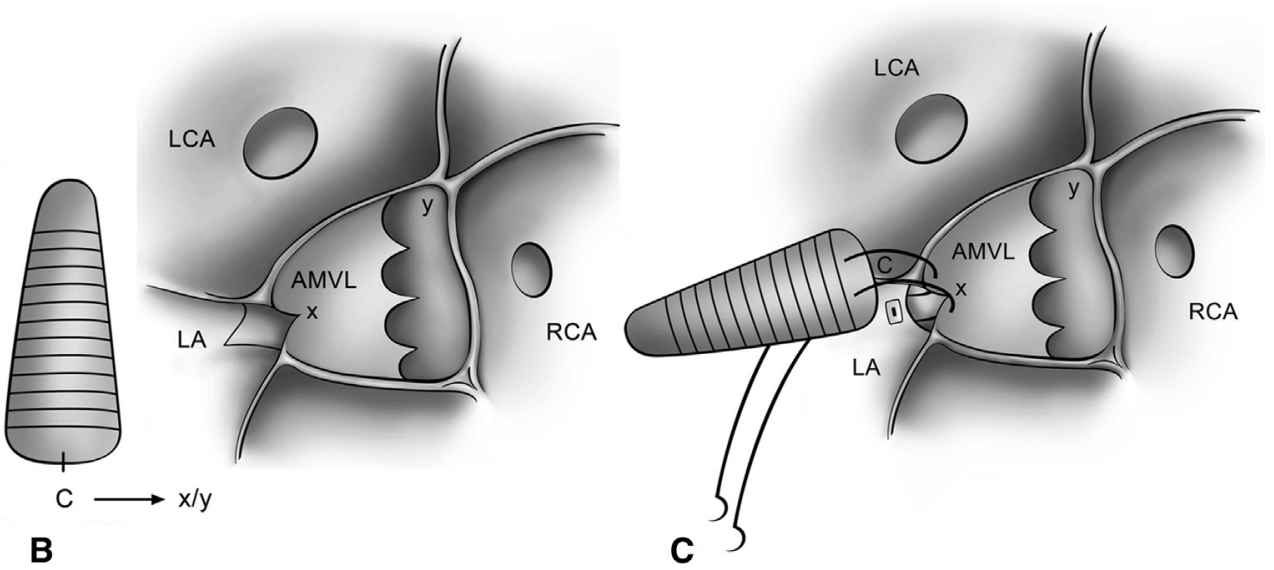

FIGURE 1. A, Incisions on the aortic root and proximal ascending aorta. B, Anterior enlargement between the right and left coronary commissures and posterior enlargement between the left and noncoronary commissures, extending sparingly into the anterior mitral leaflet ( $A M V L$ ). C, Point $C$ at patch is sutured to points $x$ and $y$, respectively, with interrupted sutures at the base of patch. Because the incision on the mitral leaflet was kept to minimum, the left atrial $(L A)$ roof could be closed with sutures incorporated into the annulus. $L C A$, Left coronary artery; $R C A$, right coronary artery.

patches are used to enlarge to aortic root anteriorly and posteriorly and are extended above the transverse aortotomy to enlarge the ascending aorta. A symmetric aortic enlargement is achieved with this 2-patch approach. By using a transverse aortotomy, the aortic annulus can be assessed satisfactorily to determine the appropriate annular enlargement strategy. Opening the aorta transversely rather than obliquely may also reduce the risk of injury to any anomalous coronary artery origin or intramural course during aortotomy. A counterincision on the aorta (Figure 1, A) is required to fit the extended patch and also to avoid kinking (Figure 2, C). Like the Yamaguchi technique, the anterior incision only traverses the annulus without entering the right ventricle or septum, thus avoiding any radical

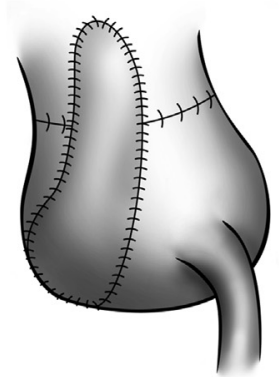

A

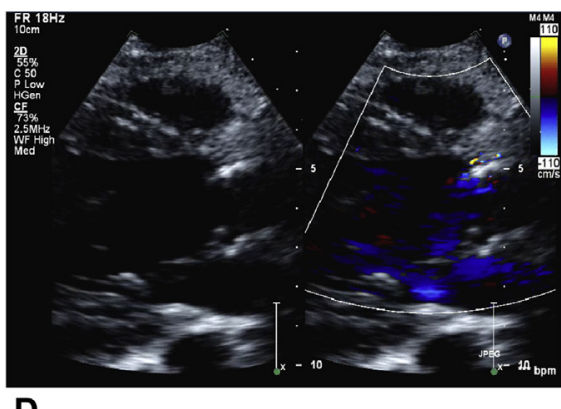

$\mathrm{R} C A$

B
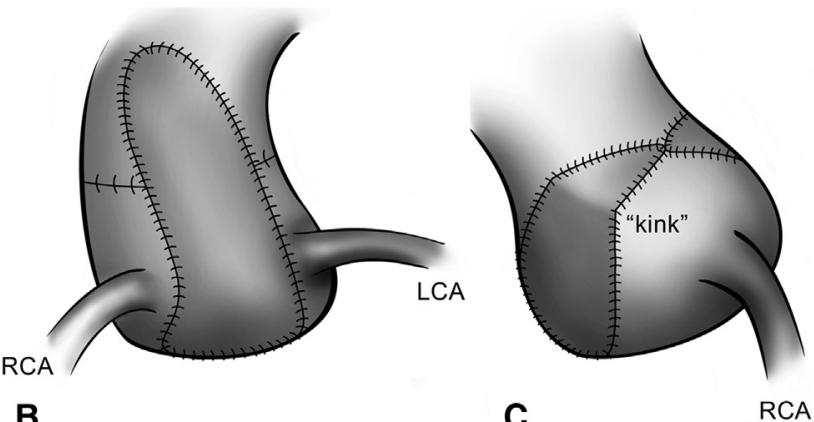

C

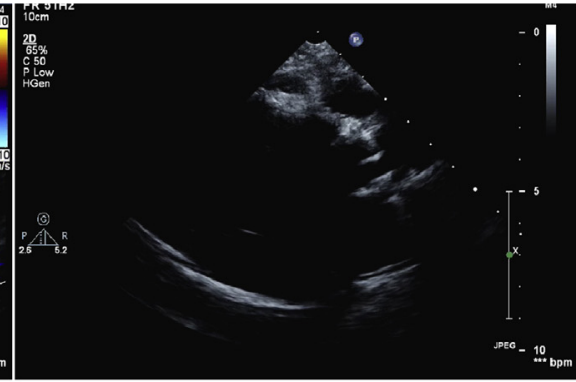

E

FIGURE 2. The rest of the patch is sutured to the aortic root with continuous sutures, with a counterincision above the aortotomy to fit the patch in the proximal ascending aorta between the left and noncoronary commissures (A) and between right and left coronary commissures (B). If the patch is sutured without counterincision, the patch will kink as illustrated (C). Postoperative echocardiography showed a peak velocity of $1.96 \mathrm{~m} / \mathrm{s}$ across the aortic valve in the first patient at 2-month follow-up (D) and a peak velocity of $1.5 \mathrm{~m} / \mathrm{s}$ in the second patient at 4-month follow-up (E). LCA, Left coronary artery; RCA, right coronary artery. 
Konno procedure. ${ }^{5}$ An additional annular enlargement anteriorly also keeps the Manouguian incision to the minimum if needed and minimizes any potential mitral valve dysfunction.

This proposed modified extended 2-patch technique enlarges the proximal aorta in 2 directions from the annulus to ascending aorta. In the context of reoperative aortic reconstruction, it can be used as an alternative to total aortic root replacement and coronary reimplantation.

We thank Ms Angela Butler for the illustrations in this article.

\section{References}

1. Vitale N, Hornung T, Ciotti G, Hamilton JR, Pozzi M, Hasan A. The Ross procedure in children under ten years of age. J Heart Valve Dis. 1999;8:601-4.

2. McBrien A, Chaudhari M, Crossland DS, Aspey H, Heads-Baister A, Griselli M et al. Single-centre experience of 101 paediatric and adult Ross procedures: mid-term results. Interact Cardiovasc Thorac Surg. 2012;14:570-4

3. Manouguian S, Seybold-Epting W. Patch enlargement of the aortic valve ring by extending the aortic incision into the anterior mitral leaflet. New operative technique. J Thorac Cardiovasc Surg. 1979;78:402-12.

4. Nicks R, Cartmill T, Bernstein L. Hypoplasia of the aortic root. The problem of aortic valve replacement. Thorax. 1970;25:339-46.

5. Yamaguchi M, Ohashi H, Imai M, Oshima Y, Hosokawa Y. Bilateral enlargement of the aortic valve ring for valve replacement in children. New operative technique. J Thorac Cardiovasc Surg. 1991;102:202-6.

\title{
Anomalous aortic origin of the coronary artery: Does pulmonary artery translocation affect coronary artery course?
}

\author{
Vitor C. Guerra, MD, ${ }^{\mathrm{a}, \mathrm{b}}$ Michael R. Recto, $\mathrm{MD},{ }^{\mathrm{a}, \mathrm{b}}$ Corey Goldman, $\mathrm{MD}, \mathrm{PhD},{ }^{\mathrm{c}}$ and \\ Thomas Yeh, Jr, MD, PhD, ${ }^{\text {a,d }}$ New Orleans, La
}

The debate around timing and choice of procedure for anomalous aortic origin of the coronary artery (AAOCA) is now the subject of an ongoing study by the Congenital Heart Surgeons Society. ${ }^{1}$ Approaches have included unroofing, reimplantation, and pulmonary artery (PA) translocation. ${ }^{2}$ Whether moving the distal PA exerts any effect on the proximal PA, and thereby acts to relieve coronary compression, has been questioned. ${ }^{2}$ Here we analyze preoperative and postoperative imaging in a single patient.

An echocardiogram performed for a murmur in a 12-year-old male football player revealed AAOCA. The patient had neither chest pain nor shortness of breath. Examination and chest radiography were unrevealing. Electrocardiography revealed normal sinus rhythm with early repolarization and increased left-sided forces. Transthoracic echocardiography (and later catheterization) revealed a 3-mm left main coronary artery (LM) arising from the right coronary sinus (Figure 1, $A l$ and $D 1$ ) that shared a common ostium with a 2-mm right coronary artery (Figure 1, Cl). The LM passed between the aorta and PA and gave rise to the left anterior descending and left

\footnotetext{
From the Tulane Pediatric Heart Center, ${ }^{a}$ the Department of Pediatrics, ${ }^{b}$ the Department of Internal Medicine, ${ }^{\mathrm{c}}$ and the Department of Surgery, ${ }^{\mathrm{d}}$ Tulane University School of Medicine, New Orleans, La.

Disclosures: Authors have nothing to disclose with regard to commercial support. Received for publication July 26, 2013; accepted for publication Aug 1, 2013; available ahead of print Sept 30, 2013.

Address for reprints: Thomas Yeh, Jr, MD, PhD, 1430 Tulane Ave, SL-22, New Orleans, LA 70112.

J Thorac Cardiovasc Surg 2013;146:1549-51

$0022-5223 / \$ 36.00$

Copyright (c) 2013 by The American Association for Thoracic Surgery

http://dx.doi.org/10.1016/j.jtcvs.2013.08.010
}

circumflex coronary arteries. There was trivial tricuspid insufficiency, no outflow tract obstruction, and normal chamber dimensions (left ventricular posterior wall, 9 $\mathrm{mm}$; septum, $11 \mathrm{~mm}$ ). Left ventricular function was hyperdynamic (shortening fraction $56 \%$, ejection fraction $86 \%$ ). Computed tomographic (CT) angiography (Figure 1, $E 1$ and F1) confirmed the diagnosis and the absence of intramural coronaries. Exercise stress echocardiography (Bruce protocol) was terminated at 10 minutes, 22 seconds for leg fatigue at 12.3 metabolic equivalents. Pulse and blood pressure increased from 83 to196 beats $/ \mathrm{min}$ (94\% of age predicted maximum) and $139 / 45$ to 204/ $41 \mathrm{~mm} \mathrm{Hg}$, respectively. There were no electrocardiographic changes, arrhythmias, or wall motion abnormalities. Threedimensional transesophageal echocardiography revealed neither coronary ostial stenosis nor intramural course (Figure 1, B1).

At surgery, little distance separated the aorta from the PA when dissected down to the coronaries to confirm their anatomy. The branch PAs were dissected to their lobar branches to relieve tension. Under bicaval cannulation with aortic crossclamping, the right $\mathrm{PA}$ was transected near its origin and its posterior wall reanastomosed anterior to the ascending aorta. The right PA was generously patched anteriorly with autologous pericardium to avoid narrowing. The patient was weaned easily from cardiopulmonary bypass with good ventricular function. Only when the PA was pressurized did we note an anterior bowing in the PA away from the aorta, undoubtedly facilitated by adventitial lysis. This accentuated arching created an obvious clear space away from the coronaries. 